\title{
Poème, paysage, esprit des lieux: Remarques sur la poésie française contemporaine
}

\section{Yves Bergeret}

omme nous arrivons aujourd'hui au milieu de l'année 1994, je vois que la poésie française connait à nouveau une remarquable vitalité.

Elle ne fut certes pas inerte ces trente dernières années; mais elle en était tout de même venue à passablement s'éreinter dans les prouesses de l'intelligence. Ces prouesses furent des acrobaties dans de petits jardins clos, sur des terrasses un peu étroites. Vollà: on avait oublié que les lecteurs de poésie sont des hommes qui ont des besoins humains, c'est-à-dire qui se posent des questions de sens, des questions sur ce qu'ils sont, sur ce qu'est ce monde, sur ce que peut maintenant, encore maintenant, être un destin.

En somme la poésie française change maintenant d'horizon. Pendant des décennies, certains de ses auteurs les plus brillants ou les plus bruyants avaient fait en sorte qu'elle se prít elle-même comme hortzon; depuis peu de temps c'est le monde des hommes qu'elle retrouve comme horzon.

Je voudrais ici être simple et indiquer en grands traits ce mouvement de vagues successives dont à mon avis résulte la poésie vivante. Cette image convient; si toutefois j'étais moins soucieux de rendre l'effet dynamique de la production poétique de ces derniers temps, je pourrais évoquer également les couches successtves ou alternées de sédimentation poétique dans la langue française.

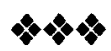

A tout seigneur tout honneur, il me faut parler d'abord de cette filiation ancienne, qui a été très forte. chargée d'un certain prestige à l'étranger et dans bien des universités américaines; il me faut évoquer ici cette filiation qui arrive au bout de ses possibilités et de sa vitalité.

Combien de poètes en effet ont brillamment, pendant deux ou trois décennies, démontré que la langue était en crise; la production de ces poètes aura été de l'ordre de la démonstration, et non pas de celui de l'intuition, de l'émotion ou de l'esthétique. Ils ont démontré avec une ferme insistance que la poésie ne pouvait plus guère avoir de sens, que le vers, le mot même, avalent enfin atteint leur incapacité à nommer, dire, porter sens. On disait tout cela de manière docte et définittve, on l'affirmait avec tous les lustres de l'intelligence empruntés ici à la linguistique, là à la psychanalyse. Le malheur, malgré tout, dans ces jeux d'esprit, c'est que nombre de ceux quil les pratiquaient portaient des jugements définitifs, sortes d'oukases contre les autres poètes, "encore" soucieux de sens. Peut-être n'était-ce là qu'un effet dévoyé de l'esprit de mai 68 , dans telle revue, dans tel comité de lecture, dans telle université de la banlieue parisienne.

A l'origine cependant de ce décapant mouvement de critique négative se place l'oeuvre de Mallarmé. On n'a cessé de la relire, de la rééditer; on n'a cessé de rêfléchir 
sur son impossibilité affichée d'aboutir au Livre et sur la "crise du vers."

Nombreux ont été ses épigones de l'après-guerre jusqu'à il y a une dizaine d'années, autour des revues qui furent fort actives comme Tel Quel, Change, et plus récemment autour de la petite revue TXT ou du groupe très resserré de la revue Action Poétique.

Mais cette ancienne avant-garde s'épuise. Elle a découragé et fait fuir ses lecteurs, de même que l’on ne peut inviter des hôtes à dîner en les contenant dans la cuisine pour uniquement voir comment c'est fait et en leur interdisant de goûter les plats dont ils ont pourtant besoin.

Il est d'allleurs amusant de constater que ces dernières années les survivants de cette famille, trop staliniens pour reconnaitre leur impasse, font semblant de déplacer le champ de leur réflexion ou le mode de leur production poétique en s'efforçant de restaurer les formes les plus fixes, voire les plus académiques de la poésie de jadis, alexandrin, sonnet, rimes, et, nec plus extra, sextine.

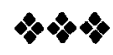

Je voudrais maintenant en venir à une seconde filiation plus vtvace. Je me demande d'ailleurs si elle n'est pas devenue inépuisable dans notre vieille civilisation occidentale. Je veux parler de la poésie du moi. Elle est presque banales, et l'on connaît bien ses divers avatars depuis la Renaissance.

Ces dernières années, je vois cette poésie séculaire du mol prospérer en se renouvelant de deux manières.

Il y a d'abord celle qui plonge intensément, presque éperdument dans la sphère intime en suivant deux grands ainés. Henri Michaux, c'est l'un deux, a somptueusement, perversement montré toutes les séductions du rêve naturel ou artificiel et sa poésie est le cri aigu que pousse le besoin de liberté du rêve: rebâtir par le jeu de métaphores du poème en prose la brûlante jubilation onirique! Antonin Artaud, c'est le second, fait du poème le cri de torture du moi dont la douleur intarissable emplit tout l'espace, celui des mots, celui du rêve, celui où le corps aurait pourtant pu agir et se mouvoir; son poème est d'abord le hurlement du moi dans le vide.

A leur suite, bien des poètes de maintenant donnent à lire, à entendre leur voix intime. Ils écrivent comme s’ils ëtaient seuls dans le monde et leurs monologues sonnent étrangement, sincèrement dans le vide. Dans le vide: dans un espace vide, pour ainsi dire peuplé de rien ni de personne, sans le moindre écho. Je voudrais citer ici Charles Juliet, affairé à ce que ses propres mots creusent sans cesse, pour la dire avec toujours plus de concision et de netteté, une sourde douleur originelle qui l'habite tout entier. Dans un style plus âpre et plus rigoureusement inégal, Marie-Claire Bancquart dit aussi la violence intérieure qui la hante. On pourrait encore évoquer l'oeuvre de Bernard Noël. Mais en somme ne pourrait-on appeler cette poésie celle du moi déchiré? Ce moi est resserré sur lui-même et la poésie lui offre l'exercice de la lucidité la plus aigüe sur ce qu'il est, c'est-à-dire une monade douloureusement, tragiquement en rupture avec son appétit de paix et d'autosuffisance.

La seconde manière de cette poésie du moi s'offre à elle-même et offre à ses 
lecteurs un horizon plus élargi que celui de la sphère intime.

Cette poésie-ci est la parole lyrique d'un mol qui se tourne vers le monde. Tout en se posant en égal de lui, il l'écoute, 11 l'interpelle, il le provoque, il le courtise, il laime, il le boude et le charme.

On reconnaît bien sûr les effets littéraires et psychologiques induits par la trilogie magnifique d'Aragon, d'Eluard et de Char. Des trois, Char est le moins connu du grand public; cependant c'est lui qui est mort le plus récemment et son influence sur la production poétique contemporaine résonne encore, même si son hermétisme irrite parfois. Ils ont tutoyé le monde. Leur audace naïve est pleine de santé.

Personne cependant, aucun poète, n'atteint actuellement leur roborative juvẻnilité dans sa production. Mais je reconnais dans l'élégie sans fin avec ses registres souples et variés telle que la pratique Lionel Ray, je reconnais dans l'élégie subtile de Richard Rognet cet appétit d'entrer en relation avec le monde.

Il y va même parfois de l'ironie: Alain Bosquet ironise, griffe, chante de désespoir devant le spectacle du monde et voudrait tant le malmener.

Qu'y a-t-il cependant de bien nouveau dans cette poésie-là? Pas grand chose, je le crains, tant commun est de chercher dialogue avec le monde, tant naturel est de chercher ce dialogue.

Mais voici maintenant une filiation beaucoup plus intéressante.

Celle-ci a su assumer la réflexion et la production critiques que Mallarmé a formulées et les transformer en les dépassant. Nulle part à l'étranger cette sublimation de la crise de la langue poétique n’a à ma connaissance donné lieu à de si intéressants développements.

C'est Yves Bonnefoy qui dès la fin des années 1950, après avoir publié des premières plaquettes marquées par un surréalisme que l'on peut sans dommage oublier, c'est Bonnefoy qui montre dans sa production combien il reste conscient de la fragilité des signes (il met en pleine lumière, en faisant de cette formule le titre de son plus beau recueil de poèmes, en 1977, que les signes se tiennent "dans le leurre du seuiln); mais cette fragilité de la langue, son statut "douteux et de plein vent," comme disait déjà Rilke, ne peut jamais faire disparaitre la soif métaphysique de l'homme; la poésie est la forme du langage qui permet le moins mal de s'interroger sur le sens de notre présence au monde et sur l'unité que nous pouvons en pressentir au delà des apparences sensibles et des signes fallacieux. A cet égard, la Leçon inaugurale que Bonnefoy a prononcée en prenant en charge la chaire d'uétudes comparées des fonctions poétiques" au Collège de France, en 1982, a fait date en réaffirmant que le poète pose à chacun et doit se poser à lui-même la question de la vérité.

De la sorte Bonnefoy a permis à la poésie française de sortir de l'impasse dans laquelle l'avant-garde l'avait enfermée.

Nombreux sont les poètes marqués par l'influence de Bonnefoy: Philippe Jaccottet, André du Bouchet, Pierre-Albert Jourdans, et bien d'autres.

Cette influence ne va toutefois pas sans quelque péril. Le souci d'approcher le 
réel pour en dégager quelque sens au delà des apparences mêlé à une rigoureuse méfiance envers le pouvoir de facile séduction des mots produit une poésie prudente; cette scrupuleuse poésie tend souvent au presque rien, au minimal, dans un style proche de la prose, presque mièvre, qui nous fait enfin, et c'était pourtant bien involontaire, revenir dans ces élégants jardinets à la française qui nous ont déjà ennuyés. En un mot, cette poésie s'expose depuis peu à un risque d'académisme.

Je voudrais toutefois attirer ici l'attention sur l'oeuvre remarquable de Monchoachi. Ce jeune poète martiniquais me parait être actuellement la voix la plus intéressante des Caraỉbes francophones; le doute sur la stabilité du signe, sur la validité de sa capacité à nommer ou décrire, ll le connaît et l'entretient dans son écriture. Mais ce doute, c'est aussi celui que le réel, vu avec la plus grande perspicacité, lui inspire: la Martinique est terre volcanique, instable; sa population est déracinée, descendante en très grande majorité d'esclaves africains; sa langue est mêlée, française imbriquée de créole; ses racines sont noueuses et rebelles: en somme la réalité même de la Martinique - et de toutes les Caraíbes - somptueuse, parfaitement moderne. est "douteuse et de plein vent" et le poème de Monchoachi est la danse magnifique dans les mots de l'instabilité du réel.

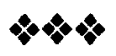

Parallèlement à la famille de poètes que je viens de décrire et dont l'ambition est d'ordre philosophique, une autre famille, fort nombreuse, prospère depuis une quinzaine d'années.

Je voudrais parler ici de la poésie du quotidien. Ses ambitions sont plus modestes. D'ailleurs le mot même d'ambition lui appartient peu. Non, il s'agit ici d'assigner à la poésie la tâche de dire, sans rhétorique ni lyrisme, la richesse de la dimension intime des choses, telles que nous les éprouvons dans la banalité au jour le jour. D’abord dire en l'aimant la grisaille quotidienne. En faire peut-être ensuite apparaitre les virtualités de mystère onirique ou même religieux. En somme ce serait un surréalisme de poche.

Ses pères spirituels sont incontestablement Jean Follain et Pierre Reverdy. On ne cesse en effet de les relire ou de se réclamer d'eux.

Le poète actuellement le plus brillant de cette famille est Jacques Réda. Il ne se fait sans doute que peu d'illusions sur sa gloire, car son influence s'explique aussi bien par ses nombreux livres que par ses fonctions de directeur de la vieille et prestigieuse Nouvelle Revue Française. Il chante en vers mélodieux et néo-classiques le charme des banlieues, les promenades à vélo, les voyages en métro.

Parmi ses confrères les plus agiles on peut citer le poète belge Guy Goffette dont les agréables plaintes élégiaques se rapprochent peu à peu de la chanson; on peut retenir Paul de Roux, poète délicat et subtil, fin lettré dont les poèmes sont en somme des aquarelles nuancées où affleure une lumière gracile et surnaturelle.

Mais sans doute le plus prometteur des nombreux poètes de cette famille est-il Dominique Pagnier. Ses derniers livres sont des recueils de poèmes en prose. Ils mettent en situation des personnages dont l'ancrage constant dans le réel est toujours accompagné d'une étrange beauté malhabile: dans cette scénographie 
baroque sans cesse en mouvement, de poème à poème, se dessine la montée ferme mais toujours retenue dans une formulation incomplète d'une lumière autre, orientale, religieuse parfois. On le voit, toute loriginalité et la force de Pagnier résident dans son amour du réel qu'll sublime en le fouillant et en le gauchissant.

Il serait incomplet de décrire ces poètes du quotidien sans présenter ceux d'entre eux pour quil le quotidien ne se comprend que dans un sens religieux, et en France, dans un sens scrupuleusement catholique. Ces poètes-ci lisent Jean Follain en même temps que Paul Claudel. Ces temps-ci leurs voix se répandent et se multiplient. On pourrait en citer plusieurs. Il suffit de nommer ici Jean-Pierre Lemaire dont la langue magnifiquement déliée et les métaphores d'une simplicité remarquablement puissante restituent de recuell en recueil les paraboles et la parole évangéliques.

\section{$\$ 4$}

Si la poésie du quotidien en est venue à dominer véritablement la production poétique française de ces dernières années, je ne pense cependant pas qu'elle en soit l'orientation la plus intéressante.

Pour ma part j'attache beaucoup plus d'attention à une autre famille d'écriture poétique et je lui prête beaucoup plus d'espoir. Cette famille ne fait pas disparaitre le moi; mais si la personne du poète ou du personnage qu'il fait parler est bien présente, ce mol n'est pas le mol lyrique qui entre diversement en résonnance affective avec son double épars dans le monde ou avec le monde lui-même. Ce moi est, en un sens, plus moderne, car le monde qui lui est socle et terreau est celui de l'Histoire. Je ne parle évidemment pas ici de la poésie engagée, vieille lune éteinte depuis des décennies. Je parle de cette parole poétique capable de faire venir dans le poème la turbulence des actions humaines, la sédimentation mouvementée des époques d'action et de pensée, la polyphonie de la parole humaine qui a sculpté et sculpte tant le paysage où nous vivons que notre demeure d'aujourd'hui. Le poème entre en résonnance avec le sens complexe du monde bâti et en interroge le sens en devenir; la réponse à son interrogation n'est jamais univoque.

Le poète qui a magnifiquement donné à la poésie française cette dimension historique moderne est André Frénaud. Il vient de mourir, au moment où l'on redécouvrait son importance majeure. Ses trois plus beaux poèmes sont "L'Etape dans la clairière," "Le silence de Genova" et surtout "La sorcière de Rome." Je voudrais que l'on relise lentement ce dernier long poème, composé de 1963 à 1969 . où, à partir des sédimentations nombreuses des civilisations qui ont bâti Rome, monte peu à peu et se dégage, se formule une parole à la fois individuelle et universelle qui interroge le ciel vide et les collines de la ville sur le sens contemporain du destin humain.

Que le poème soit ainsi en prise avec l'Histoire est, malgré tout, possible. Et peut-être bien ne faut-il pas écrire malgré, mais grâce. Grâce à Mallarmé qui nous a enseigné le doute dans le pouvoir directement nominateur du langage et nous a fait prendre conscience du vide au centre de la parole poétique: ce vide, si on y regarde maintenant bien, est non pas paralysant, mais stimulant, qu'il soit centrifuge ou centripète et Seferis dans son magnifique "Roi d'Asiné" en a parlé 
avec une intelligence et une générosité encore non dépassées. Non pas malgré, mais grâce à Bonnefoy, lui aussi.

Le poème est l'empreinte dans les mots en vers de l'esprit des lieux. J'entends par esprit des lieux la sédimentation des expériences humaines dans l'espace d'un paysage. Le poète rencontre ces lieux; le mol, par le poème, interroge la polyphonie de l'Histoire.

Si Frénaud, homme de lucidité et d'audace, poète du fragment et aussi bien du long poème presque épique, fonde en France magnifiquement ce type d'ècriture poétique, on pourrait considérer qu'il est suivi par de brillants poètes. En premier lieu par Lorand Gaspar, dont l'oeuvre poétique traverse les espaces de l'Europe et du Proche-Orient en alternant avec une rêflexion théorique d'une grande modernité sur les modes de constitution des systèmes de signes. Je voudrais également évoquer ici la recherche rigoureuse de Pierre-Alain Tâche, poète suisse. Je voudrais enfin attirer l'attention sur l'oeuvre d'un nouveau poète, Emmanuel Mosès, capable déjà d'interroger le destin dans la turbulence des signes réalistes aussi bien que culturels de la vieille Europe et du Proche-Orient, avec une vitalité remarquable.

Il va de soi que c'est cette dernlère famille de poètes, qui n'est pas actuellement la plus nombreuse, qui me paraît la plus intéressante et assurément la plus prometteuse. 